\title{
Bilateral Optic Atrophy with Thickened Meninges (Idiopathic Hyper- trophic Pachymeningitis) - A Rare Entity
}

\author{
Ravouf Asmi, D.M.,' Feroze Ahmad, M.D., ${ }^{2}$ Rangreze Imran, M.D.,' Zarka Amin Masoodi, M.D., ' \\ Mushtaq Ahmad Wani, D.M.' \\ Departments of 'Neurology and ${ }^{2}$ Internal Medicine, , Sher-i-Kashmir Institute of Medical Sciences, Soura, Srinagar India
}

\section{A B S T R A C T}

\begin{abstract}
Idiopathic hypertrophic pachymeningitis (IHP) is a rare, chronic nonspecific and granulomatous inflammatory disorder of the dura with unknown etiology. The diagnosis can be established by an open biopsy and exclusion of all other specific granulomatous and infectious diseases. We report a case of Idiopathic hypertrophic pachymeningitis who manifested primarily by headache and visual loss with optic atrophy. The patient was thoroughly investigated and showed no evidence of underlying cause. The diagnosis was established by an open biopsy. JMS $2011 ; 14(1): 25-27$
\end{abstract}

Key Words: Pachymeningitis, Tolosa Hunt syndrome, headache, granulomatous inflammatory disorder

Idiopathic cranial Hypertrophic pachymeningitis (ICHP), a rare clinical entity of diverse etiology causing thickening of the dura. When an exhaustive evaluation fails to reveal a cause, Idiopathic Hypertrophic Meningitis (IHCP) is considered.IHCP affects predominantly male patients. Diagnosis is established by dural biopsy and exclusion of other causes. Adequate therapeutic management is still a matter of debate. Several therapeutic strategies have been proposed, including steroids, immunosuppressive agents, and surgical removal of the affected tissue.

We report a case of IHCP who presented with headache and vision loss. Patient was managed with steroids and Azathioprine and is presently doing well on our follow up.

Correspondence and Reprint request to:

Dr. Ravouf Asmi

Associate Professor

Department of Neurology,

Sher-i-Kashmir Institute of Medical Sciences, Soura, Srinagar

190011, India

E-mail: ravoufasimi@yahoo.co.in

\section{Case Report}

A35 year female patient, normotensive, non smoker, non diabetic having painful eye movements and headache, was diagnosed to be having Tolosa Hunt syndrome. She was put on oral steroids and her symptoms improved. She was doing well for two months when she gradually started with painless diminition of vision and within next 2-3 months had complete loss of vision.

Patient reported to our clinic after 1 year with severe headache and loss of vision. She had stopped steroids of her own. Her systemic examination was normal but ophthalmic examination revealed bilateral optic atrophy. Her routine urine analysis, blood counts and serum chemistry were normal. The serum was negative for rheumatoid factor, antinuclear antibodies, VDRL test, hepatitis B surface antigen ( $\mathrm{HBsAg}$ ) and anti-double stranded DNA, Work up for sarcoidosis such as chest radiograph, serum calcium and angiotensin converting enzyme were negative. Bone marrow examination and ultrasound scan of the abdomen, to exclude a neoplastic and chronic inflammatory process, were normal. Cerebrospinal 
fluid (CSF) analysis revealed 7 lymphocytes $/ \mathrm{mm},{ }^{3}$ proteins of $29 \mathrm{mg} / \mathrm{dl}$ and sugar of $81 \mathrm{mg} / \mathrm{dl}$. Microbiological evaluation of the CSF for cryptococcal antigen, VDRL, and cultures for acid fast bacilli (AFB) and fungi yielded no positive results. Contrast MRI brain revealed diffuse meningeal enhancement compatible with hypertrophic pachymeningitis (Fig.1)

\section{Discussion}

The etiology of Hypertrophic chronic pachymeningitis (HCP) is unclear, although several causative factors have been recognized,including infections, autoimmune disorders, and neoplasms. ${ }^{1,2}$ However, most cases, with no evident etiology are classified as Idiopathic Hypertrophic Pachymeningitis. ${ }^{3}$ as
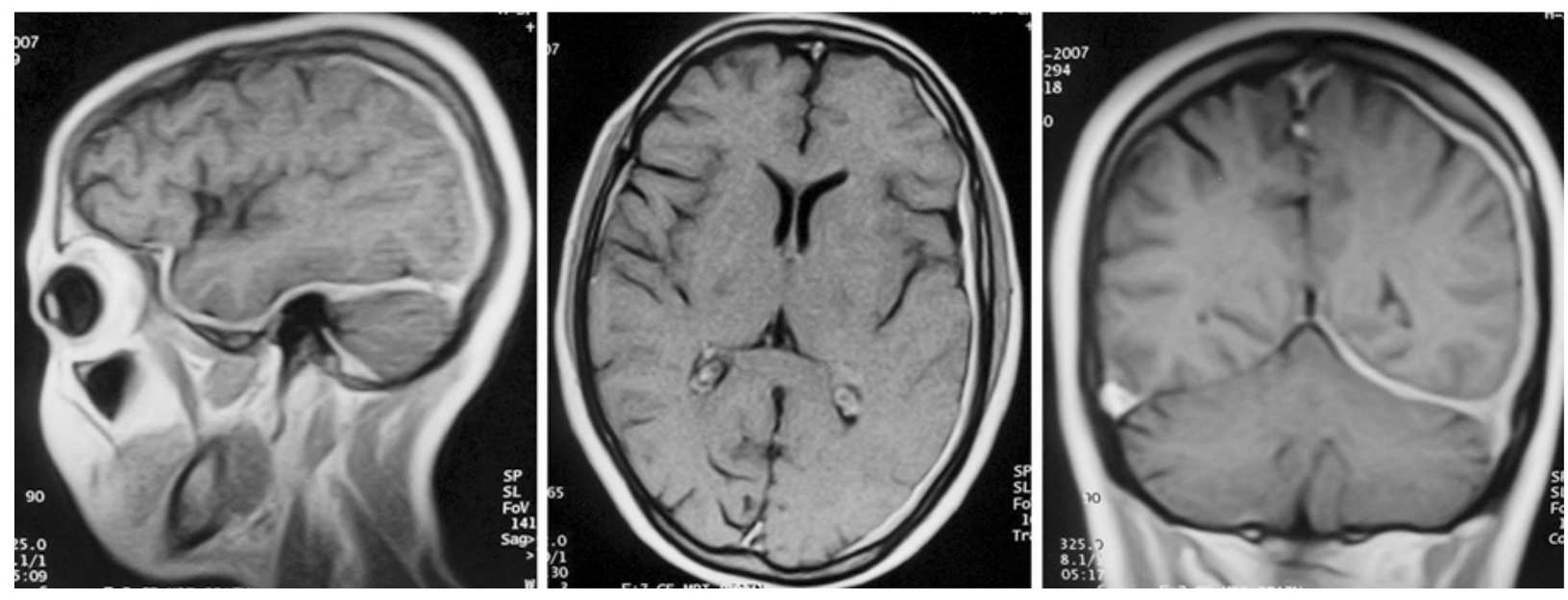

FIGURE 1: Contrast MRI revealing diffuse meningeal enhancement

Right parieto-occipital craniotomy was done, which revealed thickened, grey and grossly fibrotic dura matter. A biopsy was obtained from the right parietal dura mater. Histopathological examination of the meningeal biopsy revealed a dense fibrous tissue infiltrated with lymphocytes and polymorphs. There was no granuloma formation or vasculitis. Specific stains for fungi and acid fast bacilli were negative.(Fig. 2) Cultures of dura were negative as were serological and PCR tests.

Patient was put on steroids and Azathioprine and within 3 weeks her symptoms improved. Presently patient is on our follow up. was the case with our patient. It has a non-specific clinical picture comprising headache, vomiting, cranial nerve palsy, ataxia, raised intracranial pressure and focal neurological defecit. Masson et al. found that headache was invariably present but did not show any specific pattern, except when there was hydrocephalus. ${ }^{3}$ They also found ophthalmoplegia to be common with frequent dysfunction of optic nerve. Other symptoms include hypoacusis, tinnitus, dizziness, trigeminal neuralgia, and dysphagia. Tolosa Hunt syndrome may be a focal manifestation of hypertrophic pachymeningitis, since they share common pathological features. ${ }^{4}$ Anatomical aspects of the cavernous sinus make it more frequently affected than
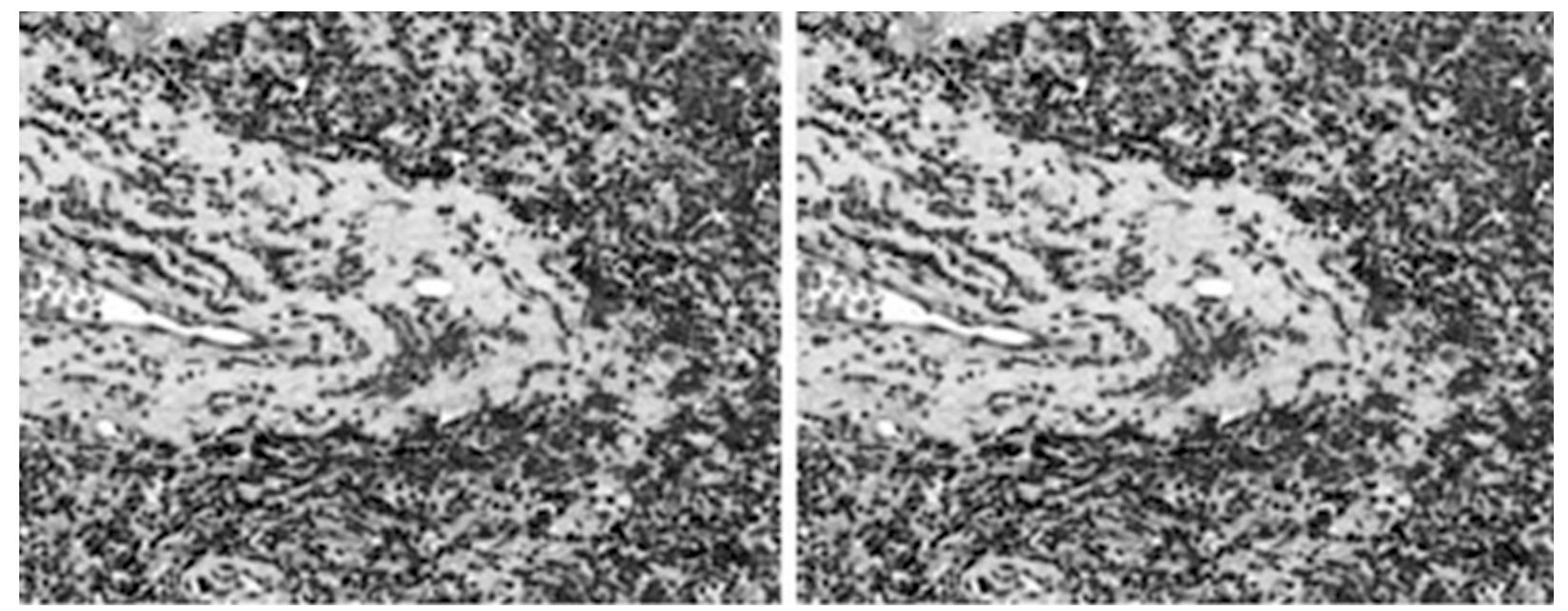

FIGURE 2: HPE of the biopsy specimen showing infiltration with lymphocytes and Polymorphs. 
other dural sinuses. ${ }^{4.5}$ The signs and symptoms in IHP are due to entrapment of cranial nerves, occlusion of CSF flow, venous sinuses and rarely arteries.

The CSF findings are dependent on the etiology. In Idiopathic cases, CSF frequently shows non-specific pleocytosis, rarely over 100 cells $/ \mathrm{mm}^{3}$, predominantly lymphocytic. There can be an increase in protein content with rise of immunoglobulins and elevation of $\operatorname{IgG}$ index. ${ }^{4} \mathrm{MRI}$ is a useful radiological method in evaluating a patient with suspected thickening of meninges. In early stage of the disease, peripheral enhancement and T2 hyperintensity are common and correspond to inflammatory activity while hypointensity is attributed to fibrosis. The use of gadolinium may differentiate between pachy- and leptomeningitis MRI also demonstrates areas suitable for biopsy and secondary lesions that may accompany the disease. ${ }^{6,7}$

Histopathological findings are that of an inflammatory process with lymphomononuclear cell infilitration and a thickened dura mater characterized by a dense hypocellular fibrous tissue. ${ }^{8}$ Specific patterns such as necrotizing vasculitis and noncaseous epithelioid granulomas can be found depending on the etiology.

The diagnosis of IHP should be made with due precaution especially in developing countries where these infections are common. In recent literature there is no consensus on the adequate management of IHCP. Spontaneous resolution of both clinical symptoms and signs, and dural thickening has been reported. ${ }^{9}$ A variety of therapeutic approaches have been tried alone or in combination. Corticosteroid therapy is often effective in ameliorating the symptoms and signs, and in stopping the disease progression. ${ }^{10}$ Attempts should be made to exclude other underlying conditions before prescribing corticosteroid or other immunosupressants. Methotrexate can be effective and a therapeutical option in patients with IHCP who are resistant to corticosteroids or present major sideeffects of chronic corticosteroids use. ${ }^{11}$

In patients with marked pleocytosis, intraventricular cytarabine has been tried with success to stop meningoproliferative process. Surgical excision is sometimes necessary for patients with mass effect due to thickening of the skull base. ${ }^{12}$ Symptomatic hydrocephalus sometimes requires ventriculoperitoneal shunting.

\section{References}

1. Masson C, Hénin D, Hauw JJ, Rey A, Raverdy P, Masson M. Cranial pachymeningitis of unknown origin: a study of seven cases. Neurology 1993;43:13291334

2. Hamilton SR, Smith $\mathrm{CH}$, Lessell S. Idiopathic hypertrophic cranial pachymeningitis. J Clin Neuro Ophthalmol 1993;13:127-134.

3. Naffziger HC, Stern WE. Chronic pachymeningitis: report of a case and review of the literature. Arch Neurol Psychiatry1949; 62:383-411.

4. Leonardo de Deus-Silva. Luciano de Souza Queiroz, Verônica de Araújo Zanardi, Enrico Ghizoni, Hoyama da Costa Pereira, George Linard Silva Malveira, Clodoaldo Pirani Jr. Benito Pereira Damasceno, Fernando Cendes. Hypertrophic PachymeningitisCase Report. Arq Neuro-Psiquiatr 2003;61:1.

5. Miwa H, Koshimura I, Mizuno Y. Recurrent cranial neuropathy as a clinical presentation of idiopathic inflam-mation of the dura mater: a possible relationship to Tolosa-Hunt syndrome and cranial pachymeningitis. J Neurol Sci 1998;154:101-105.

6. Misra UK, Kalita J. Idiopathic hypertrophic cranial pachy-meningitis: easy to suspect, difficult to prove. 2002;50(1): 4-5.

7. Bang OY, Kim DI, Yoon SR, Choi IS. Idiopathic hypertrophic pachymeningeal lesions: correlation between clinical patterns and neuroimaging characteristics.Eur Neurol 1998;39:49;49-56.

8. Friedman DP, Flanders AE. Enhanced MR imaging of hypertrophic pachymeningitis. AJR 1997;169:14251428.

9. Lam BL, Barret DA, Glaser JS, Schatz NJ, Brown HH. Visual loss from idiopathic intracranial pachymeningitis. Neurology 1994;44:694-98.

10. Tanaka M, Suda M, Ishikawa Y, Fujitake J, Fujii H, Tatsuoka Y. Idiopathic hypertrophic cranial pachymeningitis associated with hydrocephalus and myocarditis: Remarkable steroid induced remission of hypertrophic duramater. Neurology 1996; 46:554-556.

11. TT Bosman, C Simonin, D Launay, S Caron, A Destée, and L Defebvre. Idiopathic hypertrophic cranial pachymeningitis treated by oral methotrexate: a case report and review of literature. Rheumatol Int 2008 ;28(7): 713-718.

12. Takuma H, Shimada H, Inoue Y, Ishimura E, Himuro K, Miki T, Nishizawa. Hypertrophic pachymeningitis with anti-neutrophil cytoplasmic antibody (p-ANCA), and diabetes insipidus. Acta Neurol Scand 2001;104: 397-401. 